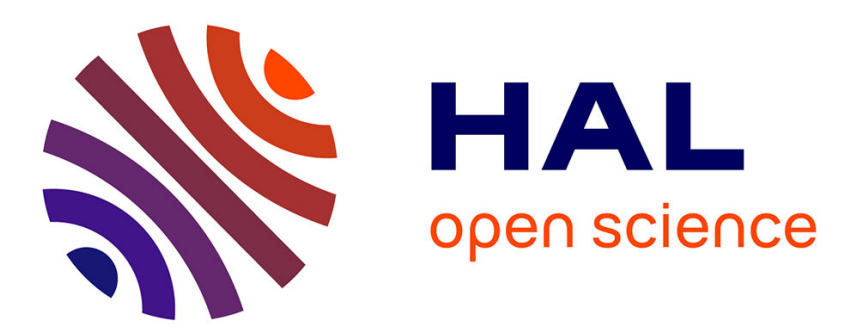

\title{
Different arrangements of simplified models to predict effective thermal conductivity of open-cell foams
}

\author{
Prashant Kumar, Frederic Topin
}

\section{To cite this version:}

Prashant Kumar, Frederic Topin. Different arrangements of simplified models to predict effective thermal conductivity of open-cell foams. Heat and Mass Transfer, 2017, 53 (8), pp.2473 - 2486. 10.1007/s00231-017-1993-8 . hal-01792097

\section{HAL Id: hal-01792097 \\ https://hal.science/hal-01792097}

Submitted on 3 Apr 2021

HAL is a multi-disciplinary open access archive for the deposit and dissemination of scientific research documents, whether they are published or not. The documents may come from teaching and research institutions in France or abroad, or from public or private research centers.
L'archive ouverte pluridisciplinaire HAL, est destinée au dépôt et à la diffusion de documents scientifiques de niveau recherche, publiés ou non, émanant des établissements d'enseignement et de recherche français ou étrangers, des laboratoires publics ou privés. 


\title{
Different arrangements of simplified models to predict effective thermal conductivity of open-cell foams \\ *Prashant $\mathrm{KUMAR}^{1}$, Frédéric TOPIN ${ }^{1}$ \\ ${ }^{1}$ IUSTI, CNRS UMR 7343, Aix-Marseille Université, Marseille, France \\ *prashant.kumar@univ-amu.fr,frederic.topin@univ-amu.fr
}

Key words: Series model, Parallel Model, Hashin-Shtrikman model, Effective medium theory, Reciprocity model

\begin{abstract}
It is often desirable to predict the effective thermal conductivity (ETC) of a homogenous material like open-cell foams based on its composition, particularly when variations in composition are expected. A combination of five fundamental simplified thermal conductivity bounds and models (Series, Parallel, Hashin-Shtrikman, Effective Medium Theory, and Reciprocity models) is proposed to predict ETC of open-cell foams. Usually, these models use a parameter as the weighted mean to account the proportion of each bound arranged in arithmetic and geometric schemes. Based on the ETC data obtained on numerous virtual Kelvin-like foam samples, the dependence of this parameter has been deduced as a function of morphology and phase thermal conductivity ratio.

Various effective thermal conductivity correlations based on material properties and foam structure valid for open-cell foams filled with any arbitrary working fluid over a solid conductivity of materials range $\left(\lambda_{s} / \lambda_{f}=10-30000\right)$ and over a large porosity range $(0.60<$ $\left.\varepsilon_{o}<0.95\right)$ are derived. Arrangement of series and parallel models together using the simplest models for both, arithmetic and geometric schemes, is found to predict excellent results among all the generic combinations.
\end{abstract}




\section{Introduction}

The thermal conductivity of porous materials, more particularly open-cell foams, plays an important role in many industrial processes. Heat conduction takes place through a solid skeleton and the fluid passing through the 3-D interconnected network of open-cells. In order to effectively utilize open-cell foams in heat transfer applications e.g. heat exchangers, volumetric solar receiver etc., accurate knowledge of their thermal transport properties are needed.

Many heat transfer mechanisms at pore scale could be included in equivalent thermal conductivity. The effective thermal conductivity refers to the case when only diffusion plays a role. The energy transport is then controlled by the effective thermal conductivity (e.g. [15]). However, at high temperatures or for foam materials of extremely low conductivity, an equivalent thermal conductivity depends also on radiative heat transfer (e.g. [6-8]) for which the expression or formulation is completely different.

The ETC of open-cell foams is not only dependent on the phases properties and porosity, but also on the structure of the materials (e.g. [4, 9-12]). Moreover, the intrinsic solid phase conductivity may be different from the parent/bulk material conductivity. Miettinen et al., [9] showed experimentally that there exists no simple relationship between intrinsic solid phase heat conductivity and porosity. Dietrich et al., [4] measured the thermal conductivity of the strut materials and obtained a decrease in their intrinsic solid phase conductivity compared to the conductivity of pure/bulk material. Kumar et al., [10-12] showed a decrease of approximately $20-25 \%$ in the solid phase thermal conductivity of bulk material when transformed in foams from their calculated ETC data.

Apart from experiments, substantial efforts to estimate the effective thermal conductivity have been made in the recent years based on: 
- $\quad$ numerical simulations using X-ray $\mu \mathrm{CT}$ images of actual samples (e.g. [13-20]),

- numerical simulations on idealized representation of open-cell foams (e.g. [10-12])

- $\quad$ empirical correlations by fitting experimental data (e.g. [1, $2,4,5,12,23,24]$

Despite the accuracy and precision of ETC results obtained from numerical simulations (or experiments); they are however considerably time and resource consuming. Therefore, their application is justified only when higher accuracy is required or, together with experimental measurements, for the validation and/or calibration of simpler models.

Moreover, ETC of a specific structure for a given working fluid has to be obtained individually on a case to case basis. Thus, an empirical correlation is a reasonable compromise between measurements and computational time for quick and accurate evaluation of ETC. It presents general applicability for a wide range of materials, porosities, and ratios of conductivities between solid and fluid phases.

Various simplified bounds and models exist in the literature to predict the ETC values for different porous media. The most common inputs for these models are material properties (e.g. solid and fluid conductivities) and material morphology (e.g. porosity). Nevertheless, prediction of ETC for different class of materials i.e. foam like structures is not straightforward. Consequently, various authors proposed empirical correlations using these bounds/models or combination between them to predict reasonable ETC values with or without an adjustable parameter depending on the ratio of constituent phases.

In open-cell foams, the empirical value of adjustable parameter in ETC correlations varies between employed models and used type of materials. Variations in adjustable parameter are due to the preferred choice of model/bound by authors and depend usually on the type of foam material used (metallic or ceramic) and thus, resulting in a massive gap to have access 
to complete range of conductivity ratios. Consequently, there is no simple model that can be used for all existing types of open-cell foams.

In this work, our intent is to develop generalized ETC correlations in accessible porosity range $\left(0.60<\varepsilon_{o}<0.98\right)$ of foams resembling Kelvin-like cell structure and extend the conductivity ratios range $\left(\lambda_{s} / \lambda_{f}=10-30000\right)$ in order to fill this gap. In this view point, five basic arrangements of simplified models and their combinations including the series and parallel models (Leach, [25]), Hashin-Shtrikman upper and lower bounds [26], Effective Medium Theory (EMT) (Landauer, [27]), and, Reciprocity model (Keller, [28]) are adapted as generic minimum and maximum bounds of thermal conductivity in both arithmetic and geometric schemes. A functional parameter is obtained by fitting ETC data obtained numerically from our previous works [10-11]. Obviously, this parameter cannot be obtained beforehand but it has been determined in the present work for the whole range of porosities and thermal conductivity ratios. Thus, it could be used as a function of the traditional input parameters (foam morphology and material property: conductivities) in order to predict ETC value for any Kelvin-like foam structure.

\section{Literature study of ETC correlations}

We discuss here ETC as an intrinsic property of conductive heat transfer and not an apparent property representing many other unspecified heat transfer phenomena (e.g. radiation, micro-convection, dispersion, evaporation-condensation etc.). Mixing heat transfer mode in a single apparent coefficient leads to a confusing situation and prohibit the comparison and use of obtained data. Nevertheless in several cases, the diffusive heat transfer is not the principal one for a given situation (e.g. [6]). This contribution could be calculated separately and added eventually. 
Usually, commercially available real foams are close to periodic in nature. Pieper and Klein [29] demonstrated that the periodic homogenization for real structures close to periodic gives very accurate results. This led researchers to derive ETC empirical correlations that are generally based either on asymptotic approaches or on a micro-structural approach in case of open-cell foams. It is widely known that the ETC strongly depends on porosity and the ratio of thermal conductivities of the constituent phases but also on a lesser extent to the distributions of the solid phase (e.g. between struts and lumps). that depends on the manufacturing process. Using analytical modelling, some authors (e.g. [1-2, 5, 14, 23, 30]) have considered lumps at the node junctions. On the other hand, others (e.g. $[4,10-12,24])$ did not assume the presence of lumps of matter at the node junctions while deriving their empirical correlations.

For the validation of empirical correlations against ETC data, it is henceforth of major importance to measure the intrinsic solid phase thermal conductivity of the foam sample because different commercially available foams employ different manufacturing techniques; that lead to significant changes in the intrinsic solid phase thermal conductivity of foams, compared to the same parent/bulk material ([4], [9-11]).

The correlations reported in the literature can be classified into three major groups: (1) obtained from fitting experimental data using simplified asymptotic bounds models (e.g. [4, 24]), (2) obtained from fitting experimental data on micro-structural foam (e.g. [1-2, 5, 12, $23,30,31]$ ), and, (3) fitting numerical simulations data on real and virtual foam structures: exploration of various geometries (e.g. [6-7,10-11, 14, 17, 18,22]). Most commonly, one or two adjustable parameters that contain information about morphology are obtained by fitting experimental or numerical ETC data to derive correlations. 
A first group of authors used only porosity and derived correlations based on series and bounds arranged in either geometric (e.g. [24]) or arithmetic (e.g. [4]) scheme. Note that these models do not introduce additional morphological parameters to describe ETC; although this influence has already been shown to be significant (see. [10-12, 17-18]). Qu et al., [30] used a so-called "morphological parameter" that was obtained by fitting the experimental data that is rather material dependent (thermal conductivity ratio) instead of foam morphology and is only valid for a few types of foam materials.

A second group of authors tried to introduce other morphological parameters in their correlation based on 2-D (e.g. [1-2]) or 3-D foam geometry (e.g. [5, 12, 23, 29, 30]) and deduced an adjustable parameter from experimental data (e.g. [1-2, 23, 30-31).. Moreover, most of these correlations are limited to very high conductive materials (e.g. Al, $\mathrm{Cu}$ ) with air or water as fluid phase and give a poor ETC estimate for foams of different materials.

For these ETC correlations for high-porosity open-cell metal foams with low conductive fluids where only parallel heat conduction through solid cell ligaments was considered $\left(\lambda_{s}{ }^{B} / \lambda_{f}>400\right)$ it was concluded that ligament cross-sectional shape do not play a strong role (at constant porosity). For low conductive foam, [12] extended the resistor model of [24] to derive ETC correlation for ceramic foams and modified the Lemlich approach. Their model could be used together to predict the intrinsic solid phase conductivity of foams of different materials irrespective of fluid phase and porosity when ETC is known.

The third of group of authors calculated ETC form numerical solution of heat equation on idealized or reconstructed open and closed-cell foams structures (.e.g. [7, 10-18, 22]). Pore scale numerical simulations (using finite element finite volume, Lattice-Boltzmann method) allow solving heat equation within both solid and fluid phases on such foam structures while varying porosity, morphology and conductivity ratios. Some of these authors further derived 
ETC correlations by introducing non-dimensional morphological parameters. [22] concluded that some simplified models could not accurately predict the ETC of foams (approximated by spherical pores, homogeneously dispersed within a solid matrix) over the complete range of porosities. [14] deduced that the realistic representations of foam structures (e.g. Cubic, Tetrakaidecahedron and Weaire-Phelan unit cells) did not account for the commercial foam irregularities and imperfections. [17] observed that using HS bounds to build correlation give the best results for most of the investigated structures. They also propose a complex arrangement of Series and Parallel models [18]. In both cases, the weighting factors can only be obtained from ETC data. [10] performed numerical simulations on Kelvin-like with convex triangular cross section ligaments to determine ETC over a wide range of solid to fluid conductivity ratios. The weighting factor appearing in their correlation is deduced from morphological parameters and ratio of solid to fluid phase thermal conductivities. This work was further extended to different strut cross-section [11]. These authors found that size of the cells struts cross section shape have negligible effects on ETC.

Various empirical ETC correlations have been developed for foams of different materials (metallic and ceramic). However, the majority of correlations concern metal foam i.e. very high conductive solid material (e.g. $[1-2,5,24])$ with high porosity $\left(0.88<\varepsilon_{\mathrm{o}}<0.97\right)$. A few are dedicated to ceramic foams (e.g. [4]) $\left(0.75<\varepsilon_{\mathrm{o}}<0.97\right)$ i.e. low conductive solid material.

Different bounds, models, adjustable and morphological parameters were used to buid correlations. No common basis could be identified among the numerous and very different proposed correlation save they all give goods results on a given set of foams and that, it is usually difficult to evaluate beforehand their applicability to a given case.

In this work, we tested different combinations of simplified bounds and models in order to retrieve their feasibility in a wide porosity range $(0.60-0.97)$, large range of conductivity 
ratios of constituent phases as well as open-cell foam samples of different materials/alloys obtained from different manufacturing processes.

\section{Simplified ETC models of porous media}

Effective thermal conductivity of open-cell foams is much higher than the granular porous media one. Since the solid has a higher conductivity, the interconnected microstructure virtually increases the pathway of thermal conduction (or energy flux) in the homogenous medium compared with the discrete granular microstructure, and therefore enhances the effective thermal conductivity of the foam material. Various effective thermal conductivity models are found in the literature; those incorporated correspond to two limiting cases for the effective conductivity, called upper and lower bounds arranged either in arithmetic or geometric schemes.

Two models of a two-phase material, which are not themselves realistic for a foam but which do provide useful results are the "parallel" and "series" models illustrated in Figure 1 (a) and (b), respectively and are often used as benchmarks for new model validations. The physical structures assumed in the derivations of the Series and Parallel models are layers of the components aligned either perpendicular or parallel to the heat flux as presented in Equations 1 and 2.

$$
\begin{aligned}
& \lambda_{\text {parallel }}=\left(1-\varepsilon_{o}\right) \lambda_{s}+\varepsilon_{o} \lambda_{f} \\
& \lambda_{\text {series }}=\frac{\lambda_{s} \cdot \lambda_{f}}{\left(1-\varepsilon_{o}\right) \lambda_{f}+\varepsilon_{o} \lambda_{s}}
\end{aligned}
$$

Hashin \& Shtrikman [26] derived effective conductivity bounds on the basis of a variational approach that were the best (i.e. narrowest) possible bounds for macroscopically homogeneous, isotropic, two-phase materials that could be derived from the components' 
volume fractions and conductivities. The bounds state that the ETC of any isotropic mixture of several isotropic conducting materials satisfies certain inequalities independently of the structure of a porous medium (see Figure 1c). The Hashin-Shtrikman (HS) bounds always lie within the Series-Parallel bounds, regardless of the components volume fractions or thermal conductivities and are given by Equations 3 and 4 .

$$
\begin{aligned}
& \lambda_{H S, \text { Upper }}=\lambda_{s}\left[\frac{2 \lambda_{s}+\lambda_{f}-2\left(\lambda_{s}-\lambda_{f}\right) \varepsilon_{o}}{2 \lambda_{s}+\lambda_{f}+\left(\lambda_{s}-\lambda_{f}\right) \varepsilon_{o}}\right] \\
& \lambda_{H S, \text { Lower }}=\lambda_{f}\left[\frac{2 \lambda_{f}+\lambda_{s}-2\left(\lambda_{f}-\lambda_{s}\right)\left(1-\varepsilon_{o}\right)}{2 \lambda_{f}+\lambda_{s}+\left(\lambda_{f}-\lambda_{s}\right)\left(1-\varepsilon_{o}\right)}\right]
\end{aligned}
$$

The problem of electrical conduction in an inhomogeneous medium could be solved by the effective medium theory (EMT). Laudauer's EMT model [27] consists of a twocomponent medium composed of two different materials, with neither phase being necessarily continuous or dispersed. The shape of each material element is assumed to be spherical. The main assumption in Landauer's theory is that the medium surrounding an element is considered homogenous, which has an effective conductivity that characterizes the overall properties of the mixture (see Figure 1d). Either component may form continuous heat conduction pathways, depending on the relative amounts of the components, therefore this structure is unbiased towards its components. Moreover, for porous media in which the solid phase forms continuous pathways, such as open-cell foams, the minimum ETC bound is expected to be given by the Effective Medium Theory (EMT) as given in Equation 5.

$$
\begin{gathered}
\lambda_{E M T}=\frac{1}{4}\left[\left(3 \varepsilon_{o}-1\right) \lambda_{f}+\left\{3\left(1-\varepsilon_{o}\right)-1\right\} \lambda_{s}+\sqrt{\left[\left(3 \varepsilon_{o}-1\right) \lambda_{f}+\left\{3\left(1-\varepsilon_{o}\right)-1\right\} \lambda_{s}\right]^{2}}\right. \\
\left.+8 \lambda_{f} \lambda_{s}\right]
\end{gathered}
$$

The reciprocity model was based on the reciprocity theorem [28] which assumes that a microstructure of two-component remains statistically equivalent when exchanging the volume fractions of the components (see Figure 1e) and is given by Equation 6. Reciprocity 
model is quite different than other models and predictions agreed well with many granular materials of spherical nature (Rio et al., [32]).

$\frac{\lambda_{R M}}{\lambda_{f}}=\frac{1+\left(\sqrt{\lambda_{f} / \lambda_{s}}-1\right) \varepsilon_{o}}{1+\left(\sqrt{\lambda_{s} / \lambda_{f}}-1\right) \varepsilon_{o}}$

\section{Comparison between experimentally and numerically calculated ETC data}

Most of the ETC data were reported in the literature for the cases where $\lambda_{s}\left(\right.$ or $\left.\lambda_{s}{ }^{B}\right) \gg$ $\lambda_{f}$ (usually metal foams). In order to perform numerical simulations to calculate ETC of foam samples, it is necessary to provide solid and fluid conductivities as an input parameters on foam structures. As discussed in sections 1 and 2, intrinsic solid phase thermal conductivity of foam materials $\left(\lambda_{s}\right)$ has not been generally measured in majority of the works. In this view point, we chose to perform numerical simulations using the experimentally measured intrinsic solid phase conductivities of $\mathrm{Al}_{2} \mathrm{O}_{3}$, Mullite and OBSiC ceramic foams $\left(\lambda_{s}=26,4.4,15\right.$ $\mathrm{Wm}^{-1} \mathrm{~K}^{-1}$ respectively) by Dietrich et al., [4]. The fluid conductivity $\left(\lambda_{f}\right)$ of air used was $0.03 \mathrm{Wm}^{-1} \mathrm{~K}^{-1}$.

As ETC is mainly porosity driven, virtual open-cell foams were generated based on total porosity $\left(\varepsilon_{t}\right)$ reported by Dietrich et al., [4]. 3-D numerical simulations at pore scale were performed on these virtual open-cell foams with circular strut cross-section in LTE condition (for detailed description, see our previous work [11]). In the Figure 2, total porosity as well as ETC data obtained numerically were compared against experimental data and are in excellent agreement (see also Table 1). However, there are very minimal differences which could be attributed to the measurements uncertainties, numerical errors and morphological disparity. This agreement confirms quantitatively and qualitatively the previously published numerical dataset of ETC, which lends confidence to develop different ETC configurations based on different simplified models.

\section{Effective thermal conductivity empirical modelling}


Depending on the availability of foam material (ceramic, alloys and pure metals), authors found out different values of adjustable parameters based on different arrangement of bounds. These adjustable parameters possessed fixed (e.g. [4, 23, 31]) and variable (e.g. [10-12, 24]) values in the most of the reported works. On the other hand, a few authors did not obtain any adjustable parameter in ETC determination for very high conductive material. The most common belief in this case is that heat conduction is mainly due to solid conductivity occurring mainly through the parallel layout while neglecting the influence of working fluid conductivity like air or water.

The present work investigates in predicting ETC values by determining a functional parameter in terms of the traditional input parameters valid for the complete range of porosities and accessible thermal conductivity ratios of Kelvin-like foams. This has been carried out by arranging various combinations of simplified bounds and models (see Equation 7 and 8).

As a first step, a generalization of the simplified model for ETC ( similar to [17]) is proposed and presented in Equatin 7. This approach is extended and applied to a geometric scheme, Equation 8 (also proposed by a few authors e.g. [10-12, 24]). The two schemes are chosen in order to identify how simple models can be best arranged based on generic minimum and maximum bounds and could predict accurate ETC values of open-cell foams.

$\lambda_{e f f}=\delta \lambda_{\min }+(1-\delta) \lambda_{\max }$

$\lambda_{\text {eff }}=\lambda_{\max } \delta^{\prime} \cdot \lambda_{\min }{ }^{1-\delta^{\prime}}$

where, $\lambda_{\max }$ and $\lambda_{\min }$ are generic maximum and minimum bounds for the ETC respectively, and $\delta$ and $\delta^{\prime}$ are the functional parameters to be determined from the fitting of various experimental/numerical data. 
Mendes et al., [17] used an adjustable parameter $\delta$ that was an explicit function of the structure of porous media and obtained by calculating ETC of solid phase alone (i.e. under vacuum condition). $\delta$ values were obtained for each case from numerical simulations by giving $\lambda_{e f f, s}$ and respective values for $\lambda_{\min }$ and $\lambda_{\max }$ under vacuum conditions.

A different strategy is applied in the current work to obtain the parameters, $\delta$ and $\delta^{\prime}$ by solving Equations 7 and 8 in terms of $\lambda_{e f f}, \lambda_{\min }$ and $\lambda_{\max }$ as:

$\delta=\frac{\lambda_{\max }-\lambda_{\text {eff }}}{\lambda_{\max }-\lambda_{\min }}$

$\delta^{\prime}=\frac{\ln \left(\lambda_{e f f}\right)-\ln \left(\lambda_{\min }\right)}{\ln \left(\lambda_{\max }\right)-\ln \left(\lambda_{\min }\right)}$

where, $0<\delta\left(\right.$ or $\left.\delta^{\prime}\right)<1$

Based on the proposed generic ETC model, given by Equations 9 and 10, and considering different possible minimum $\left(\lambda_{\min }=\lambda_{\text {series }}, \lambda_{H S, L o w e r}, \lambda_{E M T}\right.$ and $\left.\lambda_{R M}\right)$ and maximum $\left(\lambda_{\max }=\lambda_{\text {parallel }}\right.$ and $\left.\lambda_{H S, \text { Upper }}\right)$ bounds, twelve different arrangements of these models are formed (Models 1-12, six for each scheme, Eqs. 11-22 in Table 2) by selecting different expressions for $\lambda_{\max }$ and $\lambda_{\min }$. In the following sections, they are called as "model" for the sake of simplicity.

These models are presented in Table 2 where explicit expressions of $\delta$ and $\delta^{\prime}$ are provided. The dataset of 2000 numerically obtained values of ETC obtained on virtual and ideal isotropic foam structures was gathered from our previous work [11] to determine values of $\delta$ and $\delta^{\prime}$ in order to account for a wide range of porosities, different morphological parameters of foam structures as well as low to high ratios of solid to fluid conductivity ratios. Previously, porosity $\left(\varepsilon_{o}\right)$ of an idealized isotropic Kelvin-like cell was expressed as a 
function of dimensionless morphological parameters $(\psi)$ of foams of any strut cross section [10-12] (Equation 23).

Same procedure was followed and $\delta$ values for minimum and maximum bounds arranged in arithmetic scheme were plotted against $\psi$ for Models 1-6 (see Equations 11-16) based on Equation 9. It can be seen in Figure 3a and 3c corresponding to the Model $1\left(\lambda_{\text {series }}\right.$ and $\lambda_{\text {parallel }}$ bounds) and Model 3 ( $\lambda_{E M T}$ and $\lambda_{\text {parallel }}$ bounds), $\delta$ values (obtained from Equation 9 using numerical ETC values [11]) collapse very well and a unique curve is obtained as presented in Equations 24-25.

$1-\varepsilon_{o}=\psi=12 \pi \alpha_{e q}{ }^{2} \beta+\frac{32}{3} \pi \alpha_{e q}{ }^{3}$

$\delta_{1}=0.6249(1-\psi)$

$\delta_{3}=0.6213(1-\psi)$

where $\alpha_{e q}=R_{e q} / L_{c}$ (ratio of equivalent circular strut radius to node-to-node length) and $\beta=L_{s} / L_{c}$ (ratio of strut length to node-to-node length) respectively. This formulation (Equation 23) is presented in [11].

The RMSD (root-mean-square-difference) values (see Equation 26) are $0.77 \%$ and $1.93 \%$ for $\delta$ values of Models 1 and 3.

$R M S D=10^{R M S(E L O G)}-1$ with $E L O G=\log (\delta)_{c a l c}-\log (\delta)_{\text {exp }}$

In case of Model 2 ( $\lambda_{H S, L o w e r}$ and $\lambda_{H S, U p p e r}$ bounds), data of $\delta$ values fit very well (see Figure 3b). An intercept has been introduced in order to obtain the best fit of ETC data. However, no explanation is yet found for the meaning of the intercept. The models 4-6 (see Figure $3 \mathrm{~d}, 3 \mathrm{e}, 3 \mathrm{f}$ ) clearly indicate that they follow same trend with porosity but do not form unique characteristics. However, these fittings are not coherent over the entire range of porosity and thus, they are not suitable to be arranged in arithmetic scheme to predict accurate values of ETC. 
In the case of arrangements of simplified models in geometric scheme, the derivation of an empirical correlation is more difficult to obtain an excellent combination of dimensionless morphological parameters and ratio of constituent phases. The geometric scheme of series and parallel models using a weighted parameter (Model 7, Equation 17) has already been presented [10-12] and thus, this scheme is not shown here. Following their procedure, $\delta^{\prime}$ values for Models 8-12 (see Equations 18-22) are calculated (obtained from Equation 10 using numerical ETC values [11]) and further efforts have been made to obtain an accurate relation between $\delta^{\prime}$ and a combination of $\psi$ and $\lambda_{s} / \lambda_{f}$ as presented in Figure 4 . However, we could not advance to obtain any relationship between $\delta^{\prime}, \psi$ and $\lambda_{s} / \lambda_{f}$ for Models 9 and 10. The plots of $\delta_{2}{ }^{\prime}, \delta_{5}{ }^{\prime}$ and $\delta_{6}{ }^{\prime}$ are represented against $\eta=\ln \left(\psi^{2.25} \lambda_{s} / \lambda_{f}\right)$, $\eta^{\prime}=\ln \left(\psi^{1.5} \lambda_{s} / \lambda_{f}\right)$ and $\eta=\ln \left(\psi^{2.25} \lambda_{s} / \lambda_{f}\right)$ in Figures $4 \mathrm{a}, 4 \mathrm{~b}$ and $4 \mathrm{c}$ respectively. The dimensionless parameters $\eta$ (and $\eta^{\prime}$ ) are the best fitting parameters to estimate effective thermal conductivity (using Eq. 8). It can be easily observed that all the values of $\delta^{\prime}$ in relation with $\eta$ (and $\eta^{\prime}$ ) collapsed on a single curve for all the different strut shapes. The RMSD values are $1.04 \%, 1.82 \%$ and $1.6 \%$ for Models 8,11 , and 12 respectively, obtained just by replacing $\delta$ with $\delta^{\prime}$ in Equation 26. From Figure 4, numerical approximation of $\delta^{\prime}$ for Models 8, 11, and 12 are given by Equations 27-29 as:

$$
\begin{aligned}
& \delta_{2}{ }^{\prime}=-0.0028 \eta^{2}+0.0395 \eta+0.8226 \\
& \delta_{5}{ }^{\prime}=-0.005 \eta^{\prime 2}+0.0876 \eta^{\prime}+0.4914 \\
& \delta_{6}{ }^{\prime}=-0.0029 \eta^{2}+0.0458 \eta+0.759
\end{aligned}
$$

There is no physical reason to choose a quadratic polynomial function in Equations 27-29 and we do not claim any physical meaning to the curve fitting. The quadratic polynomial function is the simplest function that gives a good approximation of ETC data. 
The development of functional parameter, $\delta$ (or $\delta^{\prime}$ ) is clearly a function of foam morphology $(\psi)$ and constituent conductivities $\left(\lambda_{s} / \lambda_{f}\right)$ depending the combination of bounds and models employed. For each combination, the functional parameter is unique. Figure 5 presents a simple algorithm to predict ETC from input parameters for foam structures. Moreover, this algorithm can also be used to predict the solid phase conductivity and morphology from a known ETC value that could help in optimizing foam structure. The applicability of these functional parameters to predict ETC is validated in the next section.

\section{Comparison and validation}

ETC correlations developed in the section 4 for different arrangements and schemes are compared and validated against the experimental and numerical data reported in the literature data for foams of different materials (ceramic and metal).

\subsection{With ceramic open-cell foams}

Predicted ETC results are firstly compared and validated against experimental data reported by [4] fro ceramic foams. In their work, data containing simultaneously morphological data, intrinsic solid phase thermal conductivity and effective thermal conductivity were measured for different foam samples $\left(\mathrm{Al}_{2} \mathrm{O}_{3}\right.$, Mullite and $\left.\mathrm{OBSiC}\right)$. Values of $\delta$ and $\delta^{\prime}$ of different models are calculated using empirical correlations according to different arrangements and schemes (Models 1, 3, 8, 11 and 12) and further applied them to estimate ETC. The analytical results are presented in Table 3. From Table 3, it can be clearly observed that all these models predict excellent ETC results (see also Figure 6-left).

\subsection{With metal open-cell foams}

A few authors (e.g. [9-11]) have already highlighted the problem of non-reporting of intrinsic solid phase conductivity of most of the foams. Most commonly, the correlations that 
have been reported in the literature were based on parent/bulk solid phase conductivity. Different metal foam materials (or alloys) and their associated ETC values of various authors are presented in Table 4. However, no information regarding their intrinsic solid phase conductivity is known.

Consequently, the methodology proposed [12] has been used to determine intrinsic solid phase conductivity while using the experimental ETC data. These authors proposed the following formulation of modified Lemlich model that is valid for any arbitrary fluid as:

$\frac{\lambda_{e f f}}{\lambda_{f}}=\frac{2}{3} \cdot \frac{\lambda_{s}}{\lambda_{f}} \cdot(\psi)^{1 / F}$

where, $F=-0.004\left(\ln \left(\psi^{2} \cdot \lambda_{s} / \lambda_{f}\right)\right)^{2}+0.0593 \ln \left(\psi^{2} \cdot \lambda_{s} / \lambda_{f}\right)+0.7144$

A simple method has been described below to calculate the intrinsic solid phase conductivity $\left(\lambda_{s}\right)$ for a given $\psi, \lambda_{e f f}$ and $\lambda_{f}$.

Step I: Equation 30 can be rewritten as:

$\frac{\lambda_{e f f}}{\lambda_{f}}=\frac{2}{3} \cdot \frac{\lambda_{s}}{\lambda_{f}} \cdot(\psi)^{1 /\left[-0.004\left(\ln \left(\psi^{2} \cdot \lambda_{s} / \lambda_{f}\right)\right)^{2}+0.0593 \ln \left(\psi^{2} \cdot \lambda_{s} / \lambda_{f}\right)+0.7144\right]}$

Step II: Assign $\lambda_{s} / \lambda_{f}=K_{s}$ and $\lambda_{e f f} / \lambda_{f}=K_{e}$ and apply natural $\log$ functions to both sides:

$$
\begin{aligned}
& \ln \left(1.5 . K_{e}\right)=\ln \left(K_{s}\right)+\frac{1}{\left[n_{1}\left\{\ln \left(\psi^{2} \cdot K_{s}\right)\right\}^{2}+n_{2}\left\{\ln \left(\psi^{2} \cdot K_{s}\right)\right\}+n_{3}\right]} \cdot \ln (\psi) \\
& \ln \left(1.5 . K_{e}\right)-\ln \left(K_{s}\right)-\frac{1}{\left[n_{1}\left\{\ln \left(\psi^{2} \cdot K_{s}\right)\right\}^{2}+n_{2}\left\{\ln \left(\psi^{2} \cdot K_{s}\right)\right\}+n_{3}\right]} \cdot \ln (\psi)=0
\end{aligned}
$$

where, $n_{1}=-0.004, n_{2}=0.0593, n_{3}=0.7144$

Step III: Using iterative process, solve for $K_{s}$.

Intrinsic solid phase conductivities were calculated using Equations 31-32 for each foam material and these values were subsequently substituted in different models (Models 1, 3, 8, 11 and 12) to predict analytical ETC values (see Table 4). From Table 4, it can be prompted that the different models are consistent with each other and their uses can be combined to 
predict accurate ETC values (see also Figure 6-right). However, the predicted values underestimate the experimental ETC data which can be attributed to the formulation of [12] by which the intrinsic solid phase conductivity of a different material is under-evaluated.

\section{Conclusion}

Different arrangements of simplified models in arithmetic and geometric schemes were tried and tested to determine effective thermal conductivity of foam samples of different materials. It has been demonstrated that arrangements of different simplified models may work for one scheme but may not work for another.

The highlight of the present work is to significantly improve the notion of fixed value of adjustable parameter and thus, determining a functional parameter of coupled thermal bounds and models as a function of foam material properties and morphology. Their validity and adaptability has been shown to predict accurate ETC values. The predicted values are compared and validated against experimental data for foams of different materials in a wide porosity range.

In this view, arrangement of Parallel and Series models in both arithmetic and geometric schemes predicts the most accurate effective thermal conductivity results. Moreover, the combination of Parallel and EMT models in the arithmetic scheme as well as Parallel and Reciprocity models, and HS upper bound and Reciprocity models arranged in geometric scheme also predict accurate results.

Depending on the availability of morphological resources, any of these models can be easily used to determine effective thermal conductivity from morphology and thermal conductivity ratio. From the present results, it can be safely concluded that the proposed correlations are most suitable for evaluating the functional parameters of both schemes. 
Finally, it may be emphasized that the most remarkable feature of the proposed models lies in the fact that they allow one to quite accurately predict the effective thermal conductivity of open-cell foam structures for any working fluid.

\section{Acknowledgement}

The authors would like to thank the ANR (Agence Nationale de la Recherche) for financial support in the framework of FOAM project and all project partners for their assistance.

\section{Nomenclature}

\section{Latin symbols}

$A / R \quad$ Side length of strut shape/Radius of strut shape $\quad \mathrm{mm}$

$L_{c} \quad$ Node-to-node length $\quad \mathrm{mm}$

$L_{s} \quad$ Strut length $\mathrm{mm}$

$F \quad$ Correlation factor (Eq. 30)

$R_{e q} \quad$ Equivalent circular strut radius $\mathrm{mm}$

\section{Greek Symbols}

$\varepsilon_{o} \quad$ Open porosity

$\varepsilon_{t} \quad$ Total porosity

$\alpha_{e q} \quad$ Ratio of equivalent circular strut radius to node-to-node length

$\beta \quad$ Ratio of strut length to node-to-node length

$\delta \quad$ Adjustable parameter in arithmetic scheme (Eq. 7)

$\delta^{\prime} \quad$ Adjustable parameter in geometric scheme (Eq. 8)

$\psi \quad$ Dimensionless geometrical parameter (Eq. 23)

$\eta \quad$ Dimensionless fitting parameter (Eqs. 27 and 29)

$\eta^{\prime} \quad$ Dimensionless fitting parameter (Eq. 28)

$\lambda_{s} \quad$ Intrinsic solid phase conductivity of foam

$\lambda_{s}{ }^{B} \quad$ Solid/Bulk phase conductivity of foam material

$\lambda_{f} \quad$ Fluid phase conductivity

$\lambda_{\text {eff }} \quad$ Effective thermal conductivity

$\lambda_{\text {parallel }}$ Effective parallel thermal conductivity (Eq. 1)

$\lambda_{\text {series }} \quad$ Effective series thermal conductivity (Eq. 2)

$\lambda_{\text {HS,Upper }}$ HS upper bound thermal conductivity (Eq. 3)

$\lambda_{H S, \text { Lower }} \quad$ HS lower bound thermal conductivity (Eq. 4)

$\lambda_{E M T} \quad$ Effective medium theory thermal conductivity (Eq. 5)

$\lambda_{R M} \quad$ Reciprocity model (Eq. 6) 
ETC Effective thermal conductivity

EMT Effective medium theory

HS Hashin- Shtrikman

LBM Lattice Boltzmann method

RT Reciprocity theorem

\section{References}

[1] Calmidi, V. V., Mahajan, R. L., The effective thermal conductivity of high porosity fibrous metal foams. ASME J. Heat Transfer. 1999; 121 (2): 466-471.

[2] Bhattacharya, A., Calmidi, V.V., Mahajan, R.L., Thermophysical properties of high porosity metal foams. Int. J. Heat Mass Transfer. 2002; 45 (5): 1017-1031.

[3] Solorzano, E., Reglero, J.A., Rodriguez-Perez, M.A., Lehmhus, D., Wichmann, M. A., De Saja, J.A., An experimental study on the thermal conductivity of aluminium foams by using the transient plane source method. Int. J. Heat Mass Transfer. 2008; 51: 6259-6267.

[4] Dietrich, B., Schell, G., Bucharsky, E.C., Oberacker, R., Hoffmann, M.J., Schabel, W., Kind, M., Martin, H., Determination of the thermal properties of ceramic sponges. Int. J. Heat Mass Transfer. 2010; 53 (1): 198-205.

[5] Yang, X. H., Bai, J. X., Yan, H. B., Kuang, J. J., Lu, T. J., Kim, T., An analytical unit cell model for the effective thermal conductivity of high porosity open-cell metal foams. Transp Porous Med. 2014; 102: 403-426.

[6] Wang, M., Pan, N., Modeling and prediction of the effective thermal conductivity of random open-cell porous foams. Int. J. Heat Mass Transf. 2008; 51 (5-6): 1325-1331.

[7] Coquard, R., Rochais, D., Baillis, D., Conductive and radiative heat transfer in ceramic and metal foams at fire temperatures. Fire Technol. 2012; 48: 699-732.

[8] Mendes, M.A.A., Talukdar, P., Ray, S., Trimis, D., Detailed and simplified models for evaluation of effective thermal conductivity of open-cell porous foams at high temperatures in presence of thermal radiation, Int. J. Heat. Mass Transf. 2014; 68: 612-624.

[9] Miettinen, L., Kekalainen, P., Turpeinen, T., Hyvaluoma, J., Merikoski, J., Timonen, J., Dependence of thermal conductivity on structural parameters in porous samples. AIP Advances. 2012; 2: 012101-15.

[10] Kumar, P., Topin, F., Vicente, J., Determination of effective thermal conductivity from geometrical properties: Application to open cell foams. Int. J. Therm. Sci. 2014; 81: 13-28.

[11] Kumar, P., Topin, F., Simultaneous determination of intrinsic solid phase conductivity and effective thermal conductivity of Kelvin like foams. Appl. Therm. Eng. 2014; 71 (1): 536-547. 
[12] Kumar, P., Topin, F., The geometric and thermohydraulic characterization of ceramic foams: An analytical approach. Acta Materialia. 2014; 75: 273-286.

[13] Vicente, J., Topin, F., Daurelle, J. V., Rigollet, F., Thermal conductivity of metallic foam: Simulation on real X-ray tomographied porous medium and photothermal experiments. Proceedings of IHTC 13, Sydney, 2006.

[14] Coquard, R., Baillis, D., Numerical investigation of conductive heat transfer in highporosity foams. Acta Materialia. 2009; 57 (18): 5466-5479.

[15] Bodla, K. K., Murthy, J. Y., Garimella, S. V., Resistance network-based thermal conductivity model for metal foams. Comput. Mater. Sci. 2010; 50: 622-632.

[16] Randrianalisoa, J., Coquard, R., Baillis, D., 2013. Microscale direct calculation of solid phase conductivity of Voronoi's foams. J. Porous Media. 16 (5), 411-426.

[17] Mendes, A.A.M., Ray, S., Trimis, D., A simple and efficient method for the evaluation of effective thermal conductivity of open-cell foam-like structures. Int. J. Heat Mass Transfer. 2013; 66: 412-422.

[18] Mendes, A.A.M., Ray, S., Trimis, D., An improved model for the effective thermal conductivity of open-cell porous foams. Int. J. Heat Mass Transfer. 2014; 75: 224-230.

[19] Ranut, P., Nobile, E., Mancini, L., High resolution X-ray microtomography-based CFD simulation for the characterization of flow permeability and effective thermal conductivity of aluminum metal foams. Exp. Therm. Fluid. Sci. doi:10.1016/j.expthermflusci.2014.10.018, 2014.

[20] Wulf, R., Mendes, A.A.M., Skibina, V., Al-Zoubi, A., Trimis, D., Ray, S., Gross, U., Experimental and numerical determination of effective thermal conductivity of open cell FeCrAl-alloy metal foams. Int. J. Therm. Sci. 2014; 86: 95-103.

[21] Krishnan, S., Garimella S., Murthy, J. Y., Simulation of thermal transport in open-cell metal foams: Effects of periodic unit-cell structure. J. Heat Transfer. 2008; 130 (2): 2450324507.

[22] Druma, A., Kharil Alam, M., Druma, C., Surface area and conductivity of open-cell carbon foams. J. Miner. Mater. Characterization Eng. 2006; 5: 73-86.

[23] Boomsma, K., Poulikakos, D., On the effective thermal conductivity of a threedimensionally structured fluid-saturated metal foam. Int. J. Heat Mass Transfer. 2001, 44: 827-836.

[24] Singh, R., Kasana, H.S., Computational aspects of effective thermal conductivity of highly porous metal foams. Appl. Therm. Eng. 2004; 24: 1841-1849.

[25] Leach, A.G., The thermal conductivity of foams. I: models for heat conduction. J. Phys. D: Appl. Phys. 1993; 26: 733-739.

[26] Hashin, Z., Shtrikman, S., A variational approach to the theory of the effective magnetic permeability of multiphase materials. J. Appl. Phys. 1962; 33: 3125-3131.

[27] Landauer, R., The electrical resistance of binary metallic mixtures. J. Appl. Phys. 1952; 23 (7): 779-784.

[28] Keller, J.B., A theorem on the conductivity of a composite medium. J. Math. Phys. 1962; 5: 548-549.

[29] Pieper, M., Klein, P., Application of simple, periodic homogenization techniques to nonlinear heat conduction problems in non-periodic, porous media. Heat Mass Transfer. 2012; 48:291-300.

[30] Qu, Z. G., Wang, T. S., Tao, W. Q., Lu, T. J., A theoretical octet-truss lattice unit cell model for effective thermal conductivity of consolidated porous materials saturated with fluid. Heat Mass Transfer. 2012; 48:1385-1395

[31] Dai, Z., Nawaz, K., Park, Y., Bock, J., Jacobi, A., Correcting and extending the Boomsma-Poulikakos effective thermal conductivity model for three-dimensional, fluidsaturated metal foams. Int. Commun. Heat Mass Transf. 2010; 37 (6): 575-580. 
[32] del Rio, J.A., Zimmerman, R.W., Dawe, R.A., Formula for the conductivity of a twocomponent material based on the reciprocity theorem. Solid State Commun. 1998; 106: 183186.

[33] Takegoshi, E., Hirasawa, Y., Matsuo, J., Okui, K., A study on effective thermal conductivity of porous metals. Transactions of the Japan Society of Mechanical Engineers B. 1992; 58: 879-884.

[34] Paek, J.W., Kang, B.H., Kim, S.Y., Hyun, J.M., Effective thermal conductivity and permeability of aluminium foam materials. Int. J. Thermophysics. 2000; 21 (2): 453-464. 
Table 2. Simplified models for effective thermal conductivity, based on the generic model given by Equations 9 and 10 , obtained by selecting different thermal arrangements for $\lambda_{\min }$ and $\lambda_{\max }$.

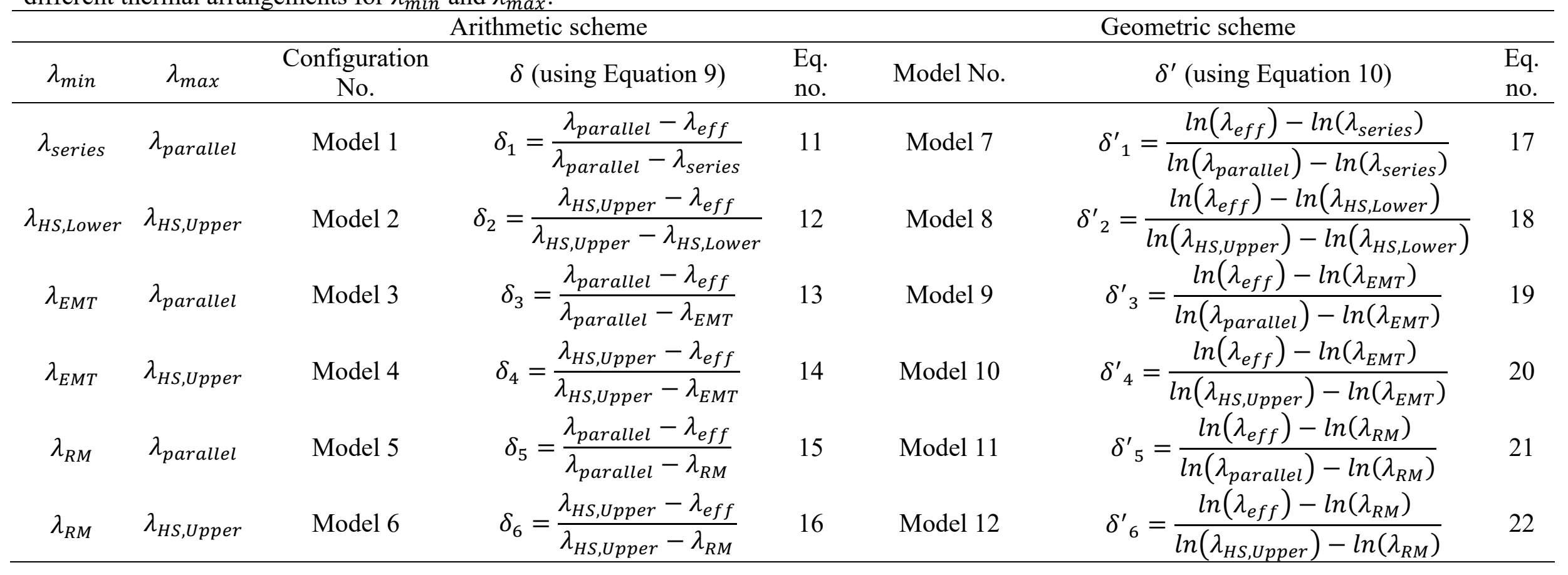




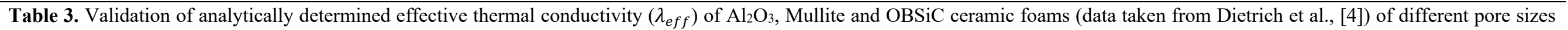
and porosities with air as an interstitial fluid.

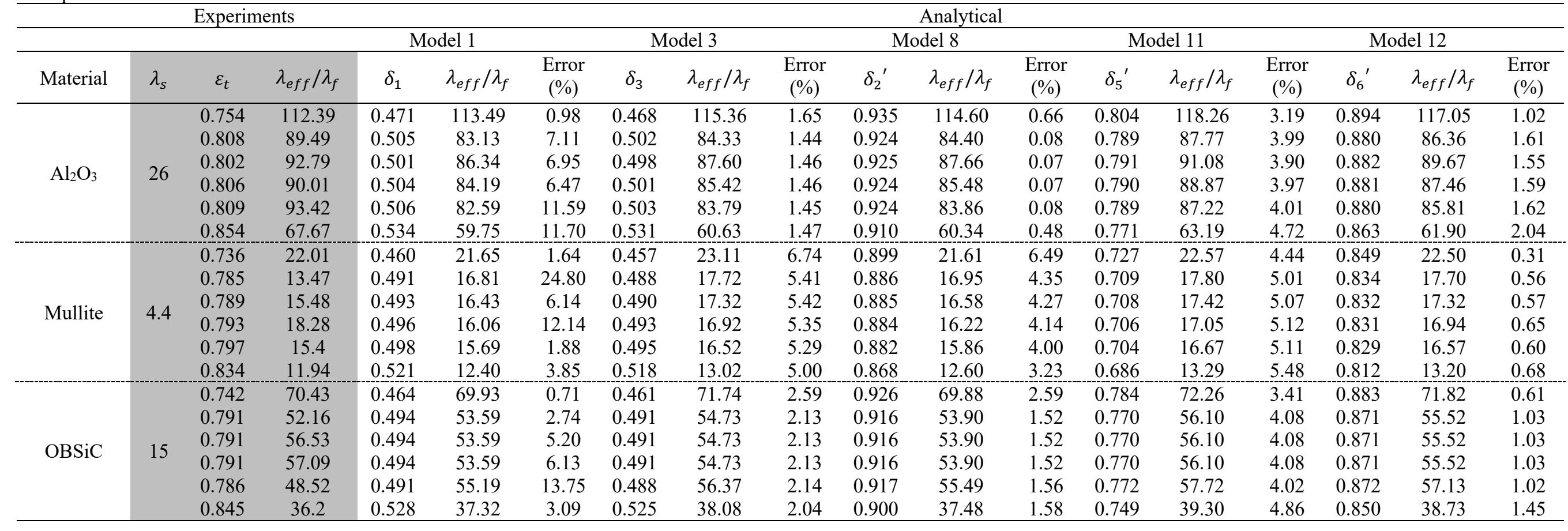

* Grey blocks represent the experimental data while the white blocks represent the calculated/analytical data. 


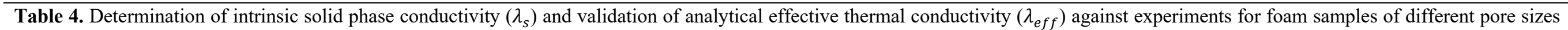
and porosities with air as interstitial fluid.

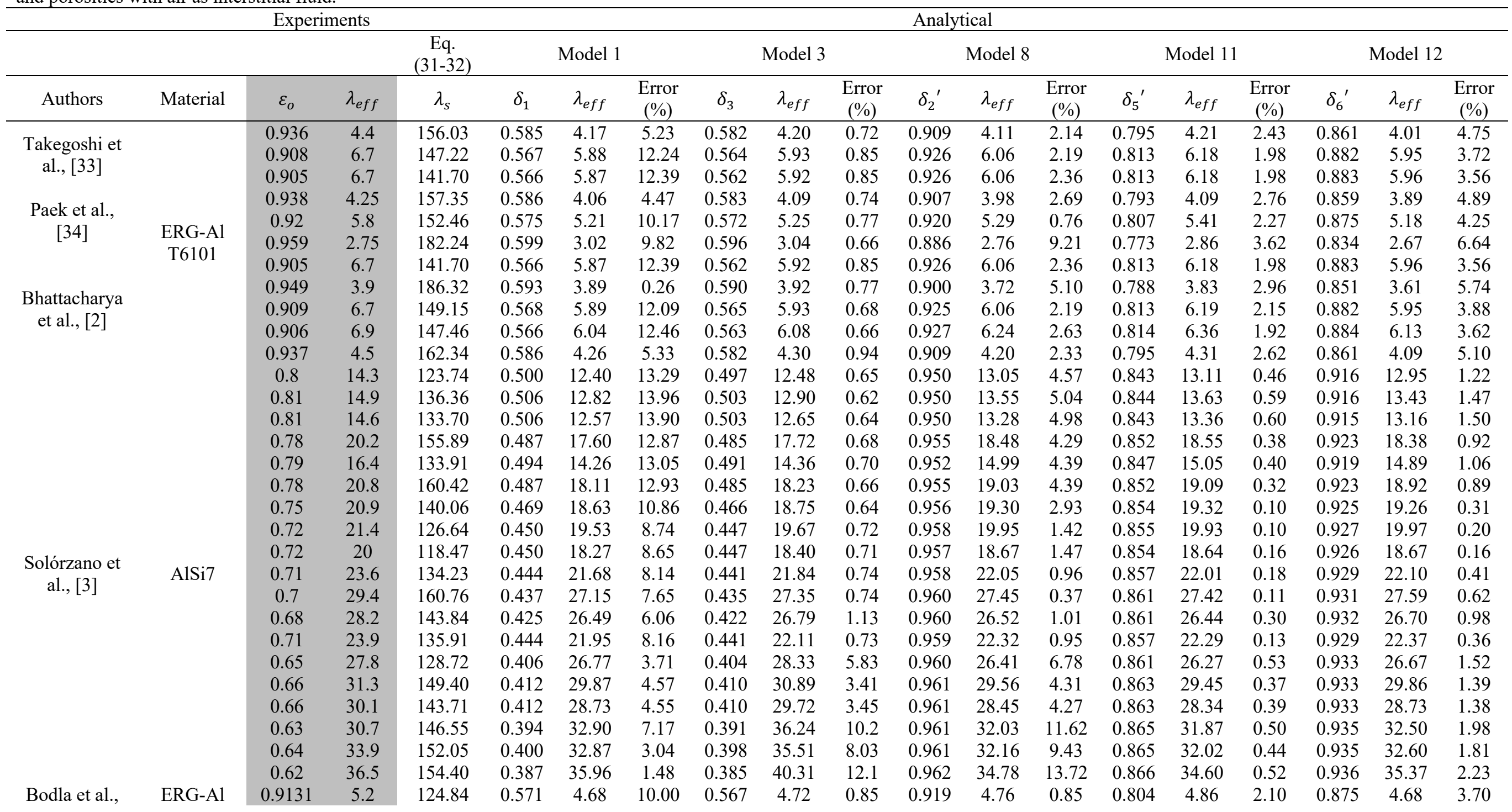

24 | P a g e 


\begin{tabular}{|c|c|c|c|c|c|c|c|c|c|c|c|c|c|c|c|c|c|c|c|}
\hline \multirow{2}{*}[15]{} & T6201 & 0.9126 & 5.21 & 124.20 & 0.570 & 4.69 & 9.98 & 0.567 & 4.73 & 0.85 & 0.920 & 4.77 & 0.85 & 0.804 & 4.86 & 1.89 & 0.875 & 4.69 & 3.50 \\
\hline & & 0.9198 & 4.52 & 121.11 & 0.575 & 4.15 & 8.19 & 0.571 & 4.19 & 0.96 & 0.915 & 4.18 & 0.24 & 0.798 & 4.26 & 1.91 & 0.869 & 4.10 & 3.76 \\
\hline \multirow{4}{*}{$\begin{array}{c}\text { Ranut et al., } \\
{[19]}\end{array}$} & \multirow{4}{*}{$\begin{array}{l}\text { ERG-Al } \\
\text { T6101 }\end{array}$} & 0.944 & 3.49 & 150.13 & 0.590 & 3.47 & 0.57 & 0.587 & 3.50 & 0.86 & 0.900 & 3.34 & 4.57 & 0.785 & 3.43 & 2.69 & 0.851 & 3.25 & 5.25 \\
\hline & & 0.927 & 4.57 & 137.25 & 0.579 & 4.24 & 7.22 & 0.576 & 4.27 & 0.71 & 0.913 & 4.23 & 0.94 & 0.798 & 4.33 & 2.36 & 0.866 & 4.15 & 4.16 \\
\hline & & 0.929 & 5.31 & 162.64 & 0.581 & 4.87 & 8.29 & 0.577 & 4.91 & 0.82 & 0.915 & 4.88 & 0.61 & 0.802 & 5.00 & 2.46 & 0.869 & 4.77 & 4.60 \\
\hline & & 0.89 & 0.372 & 6.09 & 0.556 & 0.32 & 13.98 & 0.553 & 0.33 & 3.13 & 0.848 & 0.32 & 3.03 & 0.668 & 0.32 & 0.00 & 0.788 & 0.32 & 0.00 \\
\hline \multirow{3}{*}{$\begin{array}{l}\text { Wulf et al., } \\
\text { [20] }\end{array}$} & \multirow{3}{*}{$\begin{array}{l}\mathrm{FeCrAl}- \\
\text { alloy }\end{array}$} & 0.85 & 0.415 & 4.82 & 0.531 & 0.36 & 13.25 & 0.528 & 0.37 & 2.78 & 0.864 & 0.36 & 2.70 & 0.682 & 0.36 & 0.00 & 0.807 & 0.37 & 2.78 \\
\hline & & 0.87 & 0.36 & 4.87 & 0.544 & 0.31 & 13.89 & 0.541 & 0.32 & 3.23 & 0.853 & 0.31 & 3.13 & 0.670 & 0.31 & 0.00 & 0.795 & 0.31 & 0.00 \\
\hline & & 0.9 & 0.32 & 5.76 & 0.562 & 0.27 & 15.63 & 0.559 & 0.28 & 3.70 & 0.838 & 0.27 & 3.57 & 0.655 & 0.27 & 0.00 & 0.777 & 0.27 & 0.00 \\
\hline
\end{tabular}

* Grey blocks represent the experimental data while the white blocks represent the calculated/analytical data. 


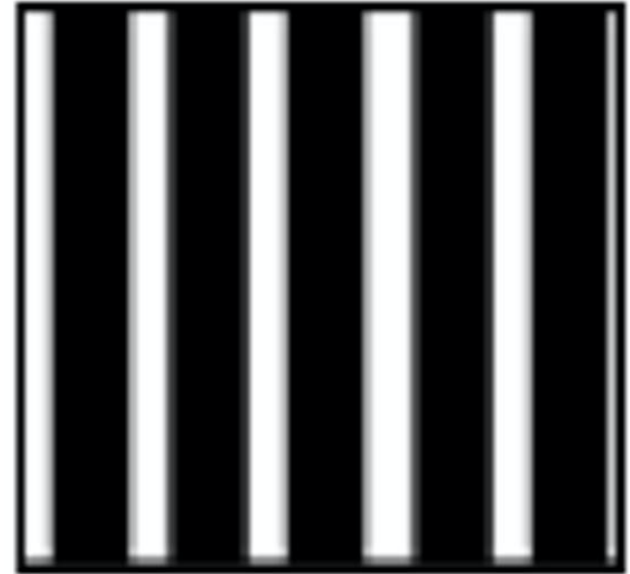

(a) Parallel Model

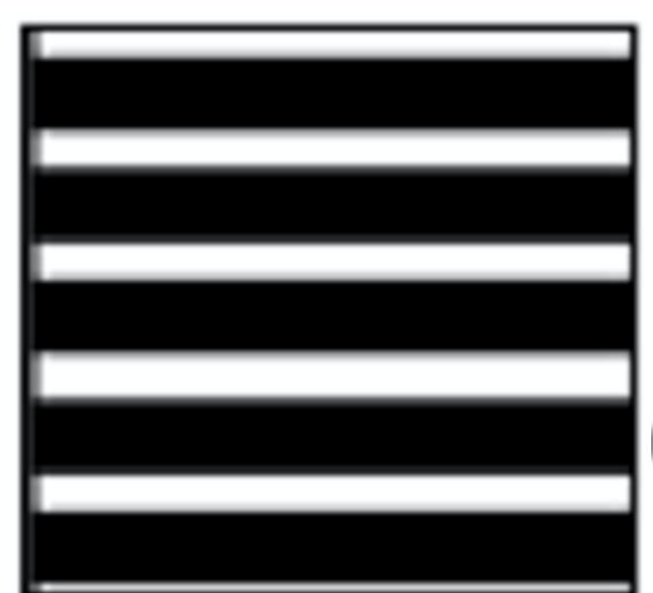

(b) Series Model

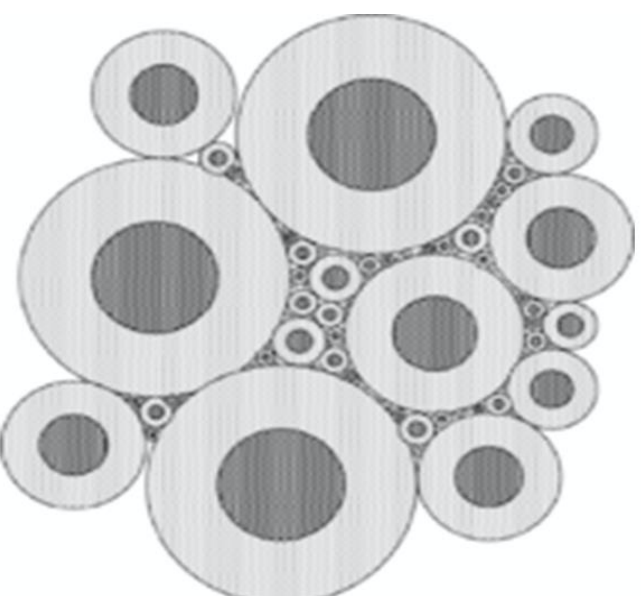

(c) Hashin-Shtrikman Model

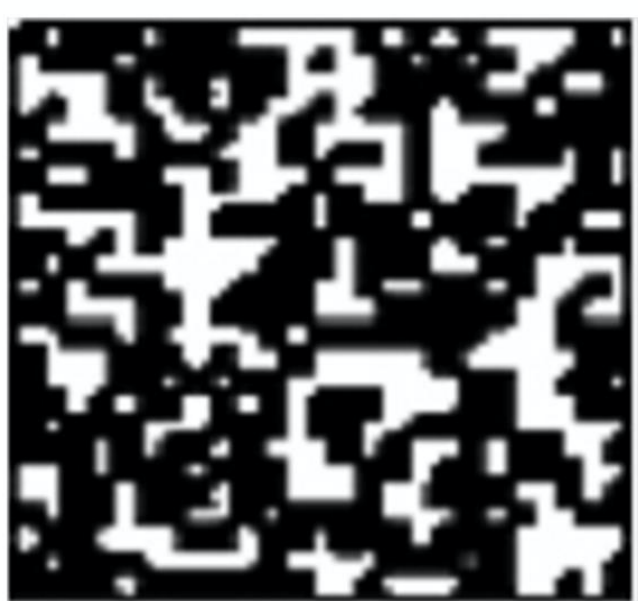

(d) EMT Model

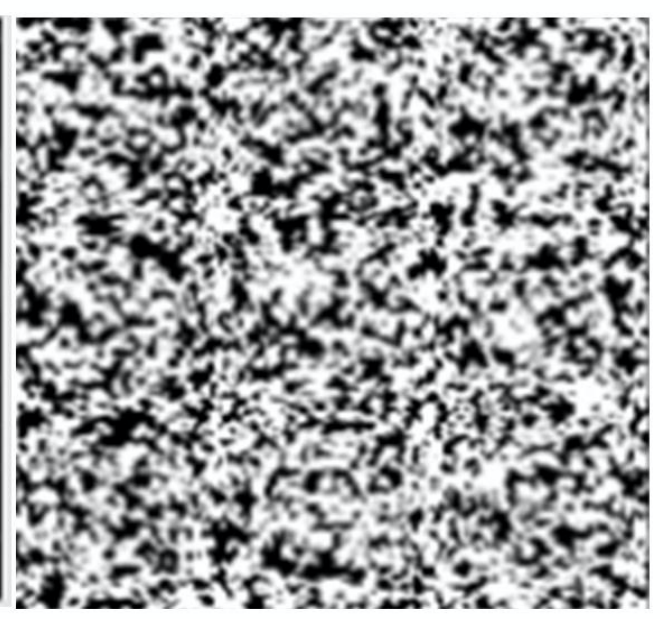

(e) Reciprocity Model

Figure 1. Five fundamental effective thermal conductivity structural models for two-component (solid and fluid phases) open-cell foam materials (assuming the heat flow is in the vertical direction). 

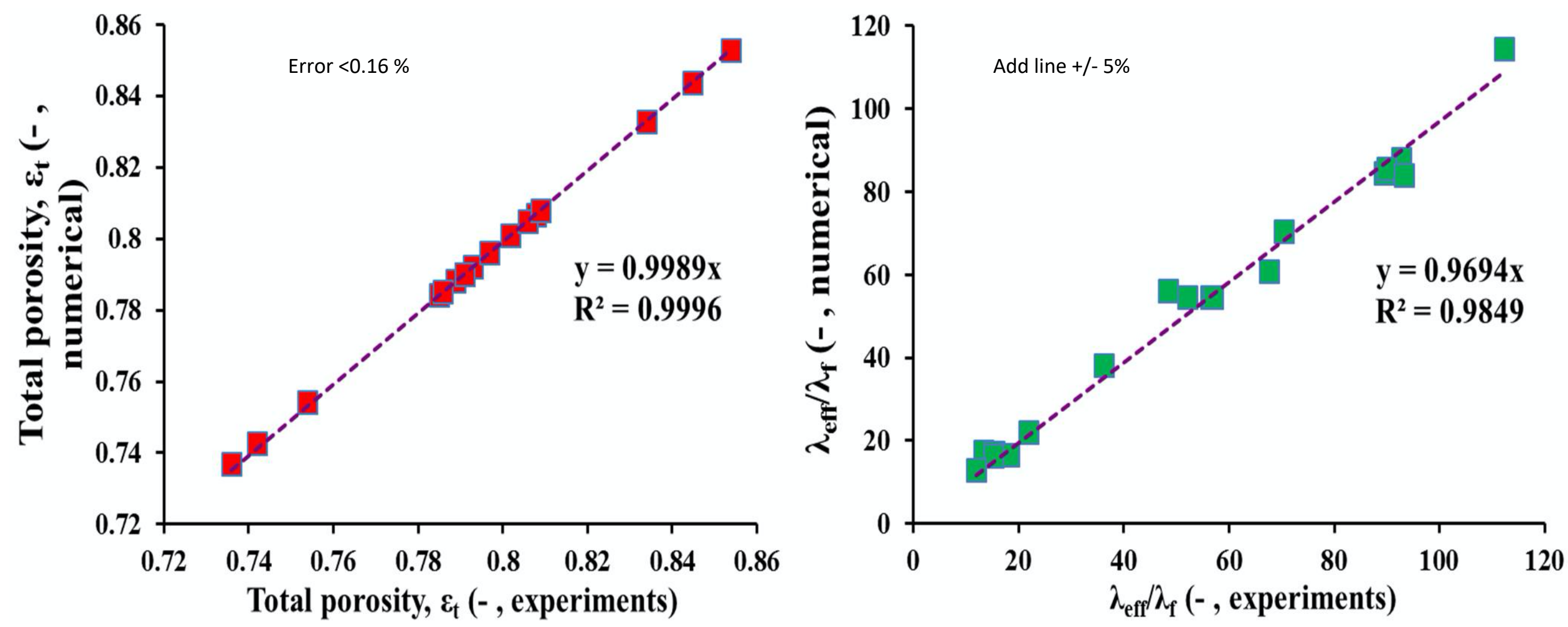

Figure 2. Comparison and validation of experimental ETC data (taken from the works of Dietrich et al., [4]) against numerically calculated ETC data of ceramic foams. 

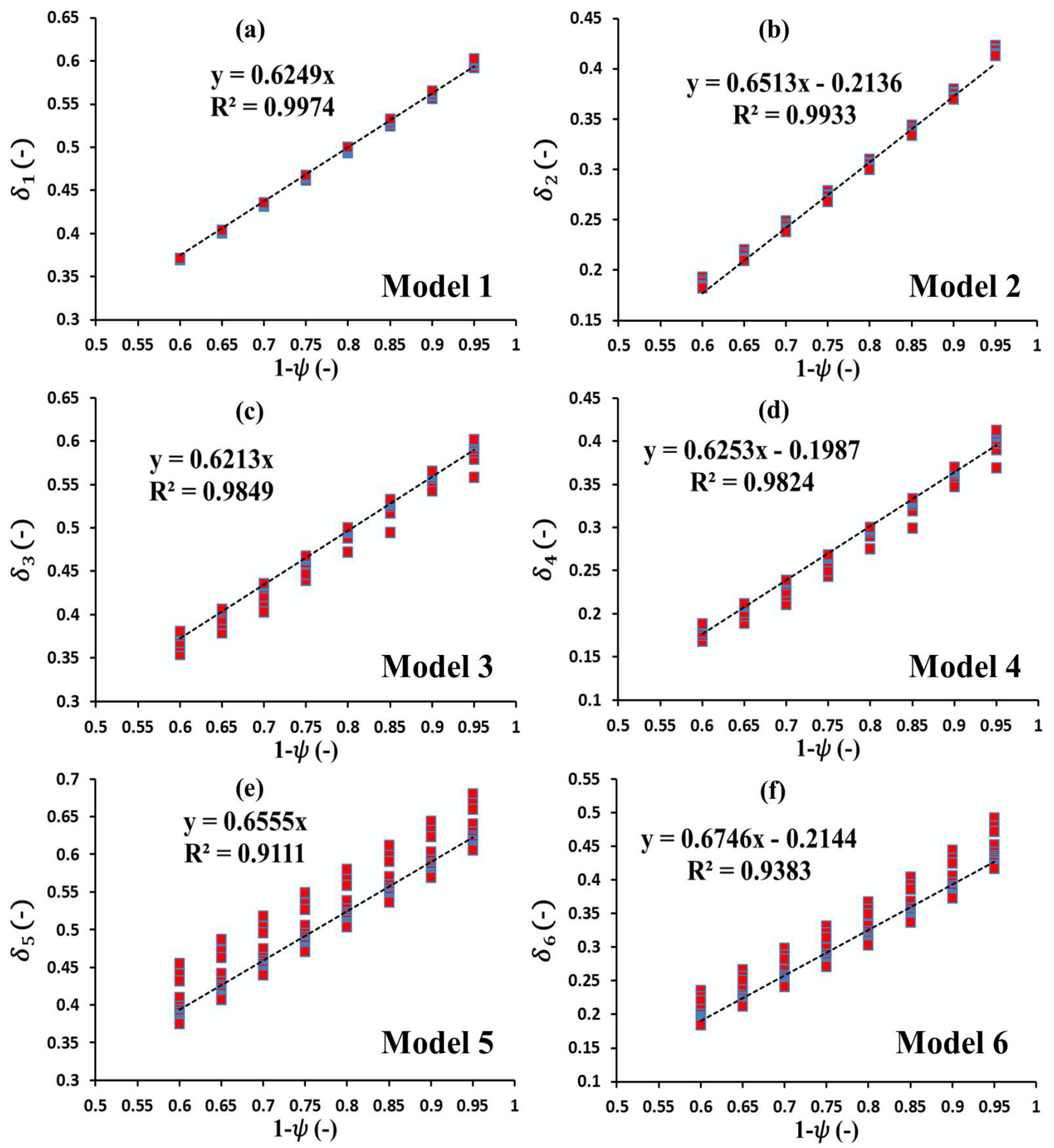

Figure 3. Plot of $\delta$ (dimensionless) for different arrangements of simplified models (Models 1-6) in arithmetic scheme. The dotted line represents the best fitting of $\delta$ values calculated using Equation 9 from numerical ETC values of Kumar and Topin [11] corresponding to different Models (1-6). Different colours of cubic data points represent $\delta$ values obtained for different porosities. 

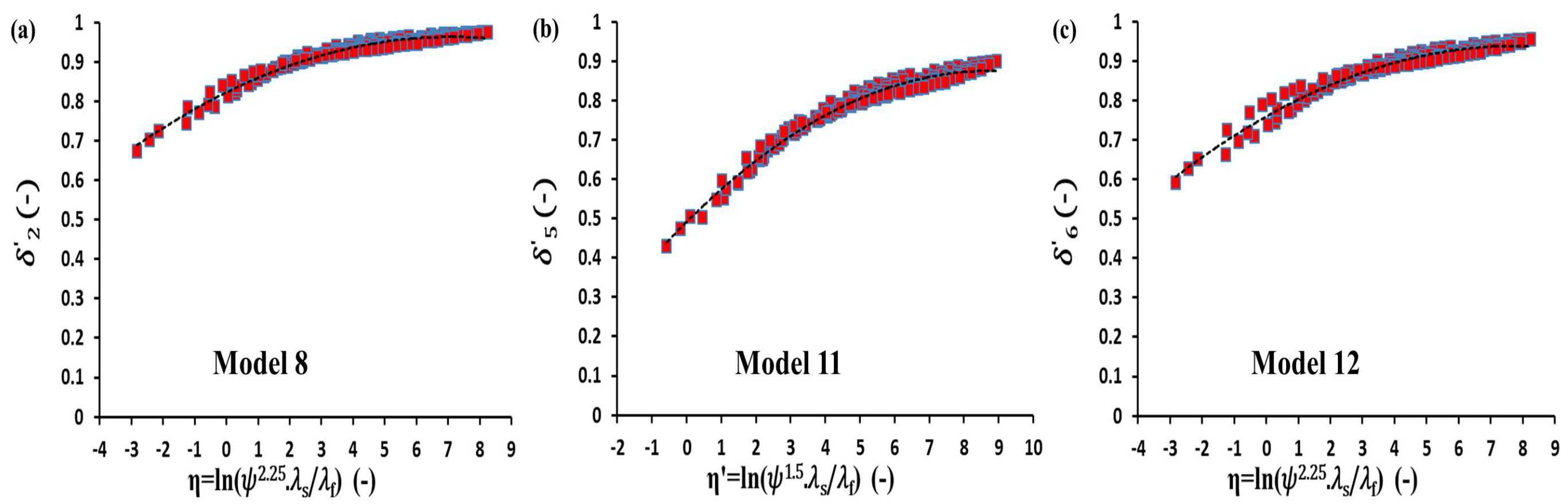

Figure 4. Plot of $\delta^{\prime}$ (dimensionless) versus fitting parameters $\eta$ and $\eta^{\prime}$ (dimensionless) of different strut shapes. (a) Model 8- Plot of $\delta_{2}{ }^{\prime}$ versus $\eta=\ln \left(\psi^{2.25} \lambda_{s} / \lambda_{f}\right)$. (b) Model 11- Plot of $\delta_{5}{ }^{\prime}$ versus $\eta^{\prime}=\ln \left(\psi^{1.5} \lambda_{s} / \lambda_{f}\right)$. (c) Model 12- Plot of $\delta_{6}{ }^{\prime}$ versus $\eta=\ln \left(\psi^{2.25} \lambda_{s} / \lambda_{f}\right)$. The errors in these models are evenly distributed. Different colours of cubic data points represent $\delta$ values obtained for different porosities. 


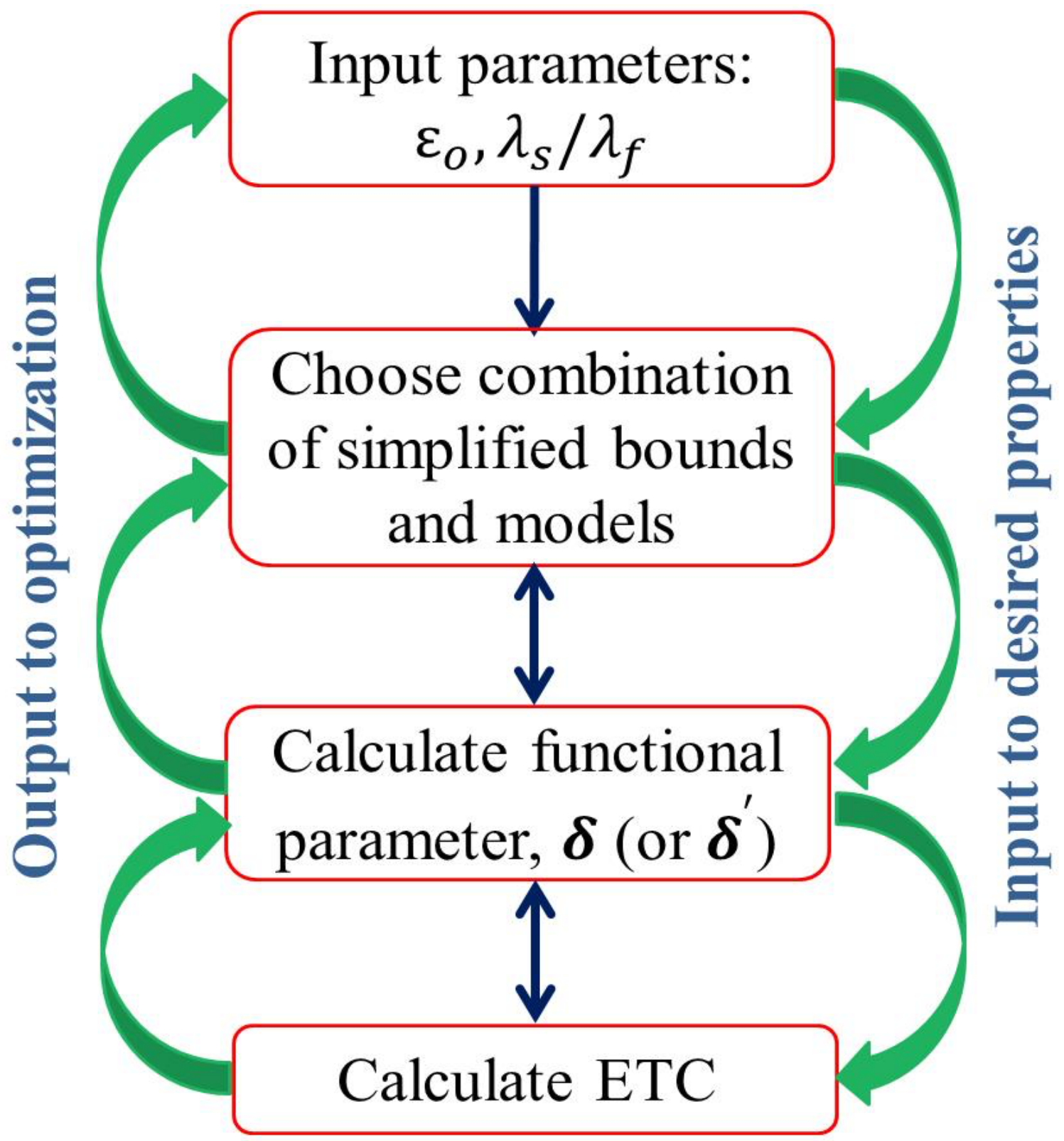

Figure 5. Algorithm to predict effective thermal conductivity (ETC) by morphological and material properties characteristics of a foam matrix. This algorithm can be used in reciprocal way- from input to output and vice versa. 

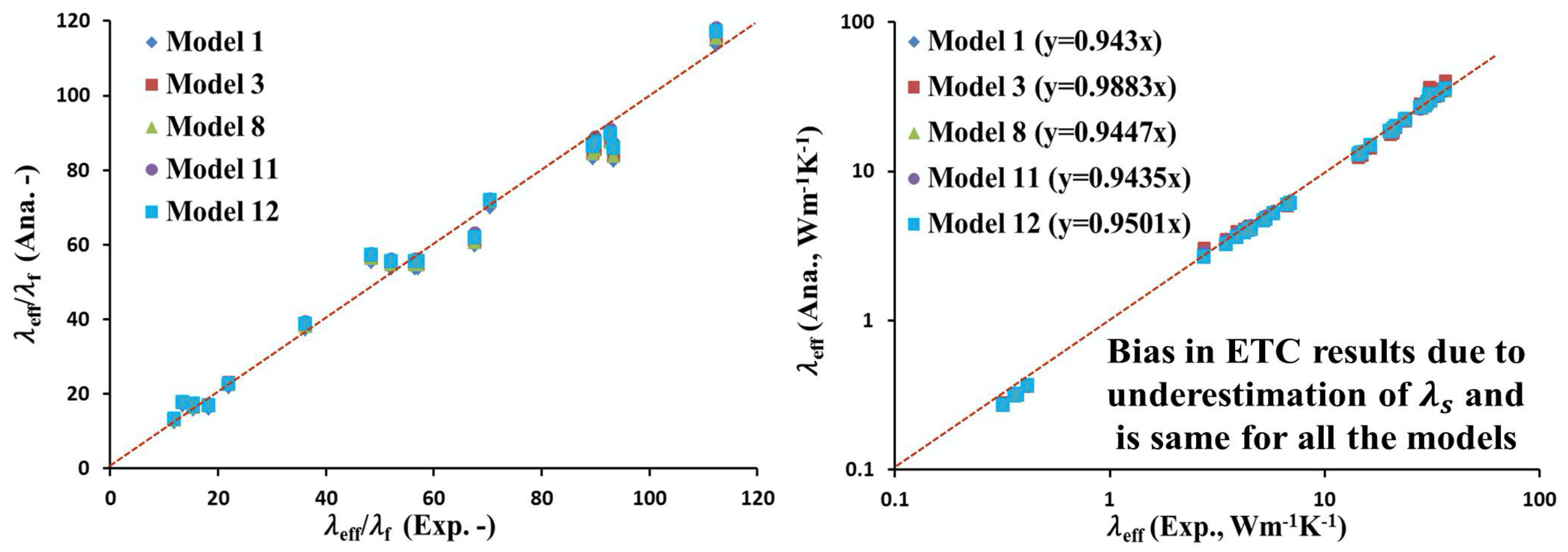

Figure 6. Comparison and Validation of experimentally and analytically obtained effective thermal conductivity $\left(\lambda_{e f f}\right)$ data by using different models (Models 1, 3, 8, 11, 12) for ceramic foams (Left) and metal foams (Right). Note that, Ana. - Analytical, Exp. - Experimental. The fitting comparison between experimental and analytical ETC values is also presented (Right). The ETC data of ceramic foams were taken from the works of Dietrich et al., [4] while ETC data of metal foams were taken from Bhattacharya et al., [2], Solórzano et al., [3], Bodla et al., [15]; Ranut et al., [19]; Wulf et al., [20]; Takegoshi et al., [33] and Paek et al., [34]. 
